Check for updates

The BMJ

Cite this as: BMJ 2021;375:n2713 http://dx.doi.org/10.1136/bmj.n2713 Published: 8 November 2021

\title{
Covid-19: Pfizer's paxlovid is $89 \%$ effective in patients at risk of serious illness, company reports
}

\section{Elisabeth Mahase}

Pfizer's oral antiviral drug paxlovid significantly reduces hospital admissions and deaths among people with covid-19 who are at high risk of severe illness, when compared with placebo, the company has reported.

The interim analysis of the phase II-III data, outlined in a press release, included 1219 adults who were enrolled by 29 September 2021. It found that, among participants who received treatments within three days of covid-19 symptoms starting, the risk of covid related hospital admission or death from any cause was $89 \%$ lower in the paxlovid group than the placebo group.

The UK government has purchased 250 ooo courses of paxlovid, which is a combination of PF-07321332 and ritonavir, alongside 480 ooo courses of another antiviral for covid-19, molnupiravir, which has now been approved by the Medicines and Healthcare Products Regulatory Agency.

Given these positive results, and after guidance from the trial's independent data monitoring committee and the US Food and Drug Administration, study enrolment has ended. A total of $70 \%$ of the 3000 planned patients from clinical trial sites across North and South America, Europe, Africa, and Asia had been enrolled, with $45 \%$ of patients located in the US.

Pfizer said it will submit its data to the FDA as soon as possible as part of its ongoing rolling submission for emergency use authorisation. The UK's MHRA will also review the drug for potential roll out this winter.

Commenting on the announcement, England's health and social care secretary, Sajid Javid, said, "If approved, this could be another significant weapon in our armoury to fight the virus alongside our vaccines and other treatments, including molnupiravir, which the UK was the first country in the world to approve this week."

In the study, trial participants were randomised 1:1, with half receiving paxlovid and the other half receiving a placebo orally every 12 hours for five days. Of those who were treated within three days of symptom onset, $0.8 \%$ (3/389) of patients who received paxlovid were admitted to hospital up to day 28 after randomisation, with no deaths. In comparison, $7 \%$ (27/385) of patients who received placebo were admitted, with seven deaths. The statistical significance of these results was reported as high $(\mathrm{P}<0.0001)$.

Similar reductions were seen in people treated within five days of symptom onset, with $1 \%(6 / 607)$ in the paxlovid group admitted up to day 28 (no deaths) and $6.7 \%(41 / 612)$ in the placebo group (10 deaths).

Overall, through to day 28 , no deaths were reported among patients who received paxlovid, while 10 people $(1.6 \%)$ in the placebo group died.

To look at safety data, the researchers included a larger group of 1881 patients in the analysis. The proportion of participants experiencing adverse events was similar, $19 \%$ in the paxlovid group and $21 \%$ in the placebo group, most of which were mild in intensity. People in the antiviral group were less likely to have a serious adverse event $(1.7 \%$ versus $6.6 \%$ in the placebo group) or to have discontinued the study because of an adverse event $(2.1 \%$ versus 4.1\%).

Mahase E. Covid-19: UK becomes first country to authorise antiviral molnupiravir. BM/2021;375:n2697. doi: 10.1136/bmj.n2697 pmid: 34737216

This article is made freely available for use in accordance with BMJ's website terms and conditions for the duration of the covid-19 pandemic or until otherwise determined by BMJ. You may use, download and print the article for any lawful, non-commercial purpose (including text and data mining) provided that all copyright notices and trade marks are retained. 